\title{
Influence of a carrier supply layer on carrier density and drift mobility of AIGaN/GaN/SiC high-electron-mobility transistors
}

\author{
M. Marso, a) J. Bernát, P. Javorka, and P. Kordoš \\ Institut für Schichten und Grenzfächen (ISG) and cni-Center of Nanoelectronic Systems for Information \\ Technology, Forschungszentrum Jülich, 52425 Jülich, Germany
}

(Received 18 November 2003; accepted 18 February 2004)

\begin{abstract}
Sheet carrier concentration and low-field drift mobility of intentionally undoped and modulation-doped $\mathrm{AlGaN} / \mathrm{GaN}$ heterostructures on $\mathrm{SiC}$ substrate were evaluated by capacitance-voltage and channel conductivity measurements. Sheet carrier concentration and average mobility at $0 \mathrm{~V}$ gate bias correspond to standard Hall results. Sheet carrier density increases from $6.8 \times 10^{12} \mathrm{~cm}^{-2}$ for the undoped sample up to $1 \times 10^{13} \mathrm{~cm}^{-2}$ for the device with the highest doping concentration, while the mobility decreases from 1800 to $1620 \mathrm{~cm}^{2} / \mathrm{V} \mathrm{s}$. The local mobility, on the other hand, depends only on the actual sheet carrier density and is not influenced by the doping concentration of the carrier supply layer. It reaches a maximum value of $2100 \mathrm{~cm}^{2} / \mathrm{V} \mathrm{s}$ at a carrier density of $3 \times 10^{12} \mathrm{~cm}^{-2}$. (C) 2004 American Institute of Physics.
\end{abstract}

[DOI: $10.1063 / 1.1704854$ ]

The polarization induced two dimensional electron gas (2DEG) at the interface of $\mathrm{AlGaN} / \mathrm{GaN}$ heterostructures allows fabrication of high-electron-mobility transistor (HEMT) layer systems and devices without the need of a carrier supply layer. HEMT devices based on undoped ${ }^{1}$ as well as modulation-doped ${ }^{2}$ layer structures were published, both with impressive dc, rf, and power performance. While the undoped devices show lower gate leakage and higher channel carrier mobility, the introduction of a carrier supply layer increases the sheet carrier density while the mobility is decreased. There is no clear evidence if the undoped or the modulation-doped structures are most suitable with respect to dc, rf, large signal and noise properties, as well as long term stability, sensitivity for trap and defect influences and the impact of a passivation layer.

First electrical evaluation of heterostructures is usually performed by standard Hall measurement, providing sheet carrier concentration and mobility. However, conventional Hall characterization only shows the mean mobility of the carriers, giving no information about the mobility distribution or the existence of a parallel conductive layer. More detailed information about the mobility spectrum can be obtained by gated $\mathrm{Hall}^{3}$ or by magnetic field dependent Hall measurements, ${ }^{4}$ or by channel conductivity measurements with HEMT devices. ${ }^{5}$ In former works these methods were used to investigate the influence of Al-content, layer quality, substrate material or temperature on channel properties. ${ }^{4-6}$ However, up to now no investigation of the influence of the carrier supply layer doping on the mobility spectrum of GaN-based HEMTs is published.

In this letter, we report on the dependence of the drift mobility on carrier concentration in undoped and modulation-doped AlGaN/GaN HEMT structures on $\mathrm{SiC}$ substrate. Investigations were performed by channel conductivity measurements on HEMTs with large (fat) gate length

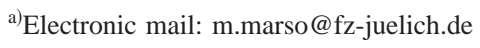

(FATFET),${ }^{5,7}$ combined with gate capacitance measurements. This method has the advantage that it uses only HEMT devices and standard measurement equipment, in contrast to the Hall based techniques. The results are compared with conventional Hall measurements and with theoretical predictions of $2 \mathrm{DEG}$ channel properties.

$\mathrm{AlGaN} / \mathrm{GaN}$ heterostructures were grown on semiinsulating $\mathrm{SiC}$ substrates by metalorganic chemical vapor deposition. Intentionally undoped structures consisted of a $30 \mathrm{~nm}$ undoped $\mathrm{AlGaN}$ layer and a $3 \mathrm{~nm}$ undoped GaN cap grown on top of a GaN buffer. Doped structures consisted of a $10 \mathrm{~nm}$ undoped AlGaN spacer, a 10-nm-thick Si-doped $\mathrm{AlGaN}$ carrier supply layer, a $5 \mathrm{~nm}$ undoped $\mathrm{AlGaN}$ barrier layer and a $3 \mathrm{~nm}$ undoped GaN cap, grown on top of a GaN buffer. The doping level $\mathrm{N}_{\mathrm{D}}$ of the carrier supply layer was $2 \times 10^{18}$ and $5 \times 10^{18} \mathrm{~cm}^{-3}$, calibrated by AlGaN test structures. AlGaN layers have an $\mathrm{Al}$ content of $\mathrm{x}_{\mathrm{AlN}} \cong 0.28$. Processing of the unpassivated devices consisted of conventional HEMT fabrication technology. As first step a mesa insulation was performed by Ar sputtering, followed by ohmic metallization $(\mathrm{Ti} / \mathrm{Al} / \mathrm{Ni} / \mathrm{Au})$ with annealing at $900{ }^{\circ} \mathrm{C}$. The gates were fabricated by electron beam lithography, with $\mathrm{Ni} / \mathrm{Au}$ Schottky contacts. Van der Pauw patterns with an active area of $0.3 \times 0.3 \mathrm{~mm}^{2}$ were processed simultaneously with HEMT devices. dc and rf results of the HEMT devices are presented elsewhere. ${ }^{8}$

Room temperature Hall effect measurements on the van der Pauw patterns (Table I) show the expected dependence on the carrier supply doping level. The sheet carrier density $\mathrm{n}_{\text {Hall }}$ increases with increasing doping level while the mobility $\mu_{\text {Hall }}$ is reduced. Channel conductivity measurements were performed on HEMTs with large gates (FATFET) to reduce the influence of the source and drain resistance. Gate length $L_{\mathrm{G}}$ and channel width $W_{\mathrm{CH}}$ were $50 \mu \mathrm{m}$ and $120 \mu \mathrm{m}$, respectively. The series resistance $\left(R_{\text {source }}+R_{\text {drain }}\right)$ was determined on HEMT devices with $2 \mu \mathrm{m}$ gate length, using the charge control model. ${ }^{9,10}$ It was always less than $10 \%$ of the FATFET channel resistance. The carrier density $n_{\text {sheet }}$ as 


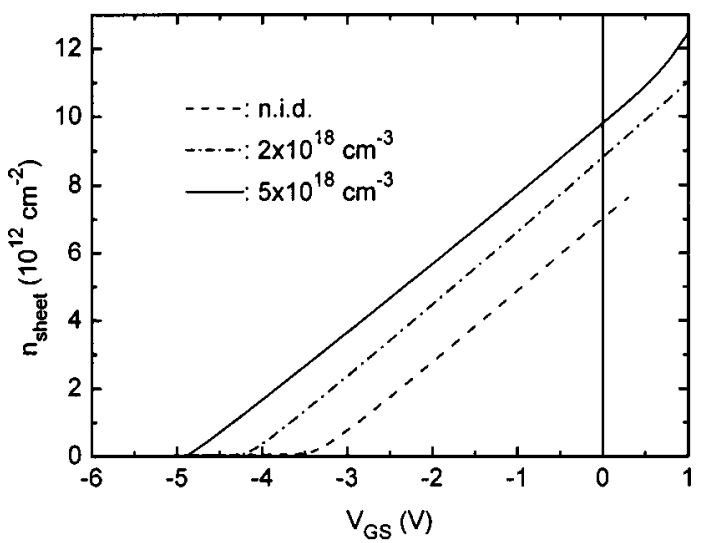

FIG. 1. Sheet carrier density as a function of the gate voltage, evaluated by capacitance-voltage measurements.

function of the gate voltage was extracted by integration of the capacitance-voltage curve of the gate Schottky electrode (Fig. 1). The average drift mobility was calculated using $\mu_{\mathrm{AVE}}=\left(G_{\mathrm{CH}} \times L_{\mathrm{G}}\right) /\left(q \times W_{\mathrm{CH}} \times n_{\text {sheet }}\right)$, where $G_{\mathrm{CH}}$ is the channel conductance at low drain voltage $(0.1-0.3 \mathrm{~V})$, corrected for the series source and drain resistance. Evaluation of the local mobility $\mu_{\mathrm{LOC}}$, i.e., the mobility of the carriers added with an increase of the gate voltage is performed by the relationship: ${ }^{11}$

$$
\mu_{\mathrm{LOC}}=L_{G}^{2} \times \Delta G_{\mathrm{CH}} /\left(C_{\mathrm{GS}} \times \Delta V_{\mathrm{GS}}\right) .
$$

Here $\Delta G_{\mathrm{CH}}$ is the change of the channel conductance for a change $\Delta V_{\mathrm{GS}}$ of the gate voltage, and $C_{\mathrm{GS}}$ is the gate capacitance.

Table I compares the Hall results with sheet carrier density and mobility evaluated by the channel conductivity method at $0 \mathrm{~V}$ gate voltage. Because the standard Hall measurement provides the mean value of the Hall mobility we compare it with the average mobility by the channel conductivity method. The sheet carrier concentration is nearly identical for both methods, while the Hall mobility $\mu_{\text {Hall }}$ has a $3 \%-7 \%$ larger value than the average drift mobility $\mu_{\mathrm{AVE}}$. This difference can be explained by the Hall factor that describes the relation between Hall and drift mobility. The theoretical value of the Hall factor ${ }^{12}$ for the investigated layer structure is in the range 1.03 to 1.10 .

Figure 2 depicts local and average drift mobility of the investigated samples as function of the gate voltage. The characteristics of the three samples have similar shapes, only shifted by the differences of the threshold voltages of the corresponding HEMT devices. The local mobility has a maximum of about $2100 \mathrm{~cm}^{2} / \mathrm{V} \mathrm{s}$, independent on the doping concentration of the carrier supply layer. The $2 \times 10^{18}$ and $5 \times 10^{18} \mathrm{~cm}^{-3}$ structures show a faster decrease of the local

TABLE I. Electron mobility and sheet carrier density of AlGaN/GaN heterostructures on $\mathrm{SiC}$ substrate, determined by Hall measurements $\left(n_{\mathrm{Hall}}\right.$, $\left.\mu_{\text {Hall }}\right)$ and by channel conductivity evaluation $\left(n_{\text {sheet }}, \mu_{\mathrm{AVE}}\right)$.

\begin{tabular}{ccccc}
\hline \hline $\begin{array}{c}N_{D} \\
\left(10^{18} \mathrm{~cm}^{-3}\right)\end{array}$ & $\begin{array}{c}n_{\text {Hall }} \\
\left(10^{12} \mathrm{~cm}^{-2}\right)\end{array}$ & $\begin{array}{c}\mu_{\text {Hall }} \\
\left(\mathrm{cm}^{2} / \mathrm{V} \mathrm{s}\right)\end{array}$ & $\begin{array}{c}n_{\text {sheet }}(0 \mathrm{~V}) \\
\left(10^{12} \mathrm{~cm}^{-2}\right)\end{array}$ & $\begin{array}{c}\mu_{\mathrm{AVE}}(0 \mathrm{~V}) \\
\left(\mathrm{cm}^{2} / \mathrm{V} \mathrm{s}\right)\end{array}$ \\
\hline Undoped & 6.95 & 1930 & 6.8 & 1800 \\
2 & 8.2 & 1780 & 8.7 & 1670 \\
5 & 9.7 & 1670 & 10 & 1620 \\
\hline \hline
\end{tabular}
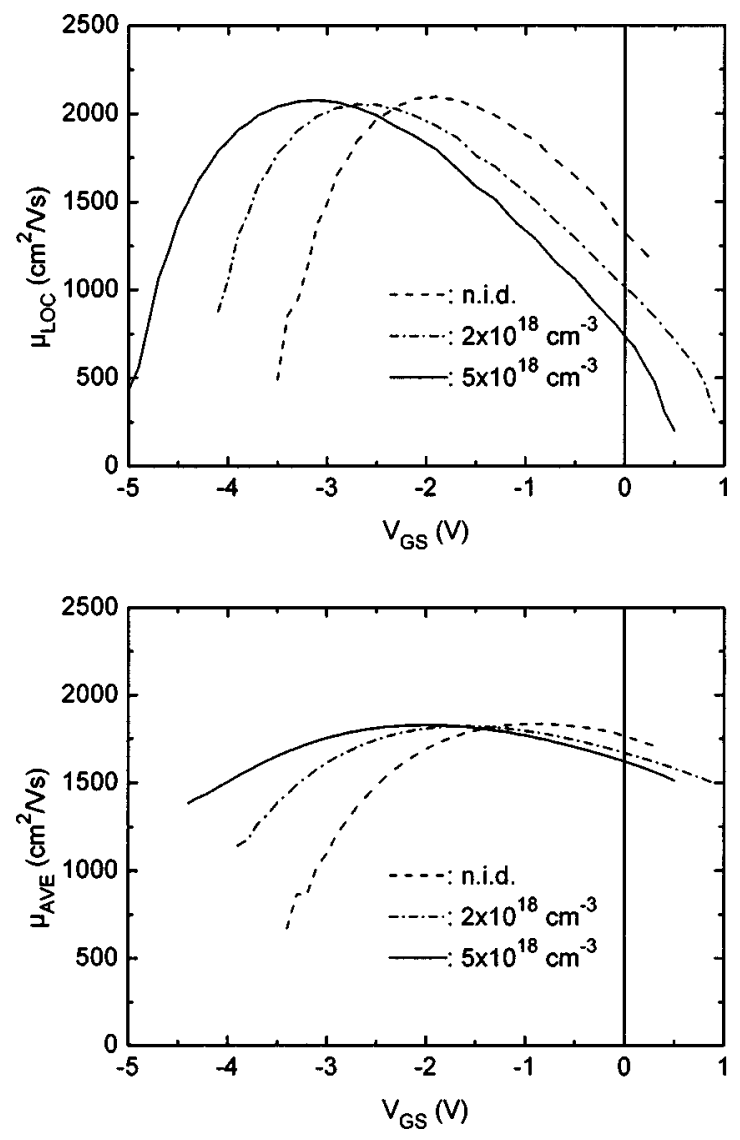

FIG. 2. Local and average drift mobility in intentionally undoped and doped $\mathrm{AlGaN} / \mathrm{GaN}$ heterostructures as a function of the gate voltage. Evaluation is performed at $V_{\mathrm{DS}}=0.3 \mathrm{~V}$ by channel conductivity method.

mobility above 0.7 and $0.3 \mathrm{~V}$, respectively. This decrease can be attributed to spill-over of electrons from the 2DEG channel to a parallel low mobility channel formed by the carrier supply layer. The increase of the gate capacitance that occurs when the gate depletion region shifts closer to surface is correlated to the fast mobility decrease, confirming our assumption.

Figure 3 shows the local drift mobility as a function of the sheet carrier density. Obviously the characteristics do not depend on the carrier supply doping beside that the doped structures can be biased to larger sheet concentration. With increasing sheet concentration the local mobility increases up

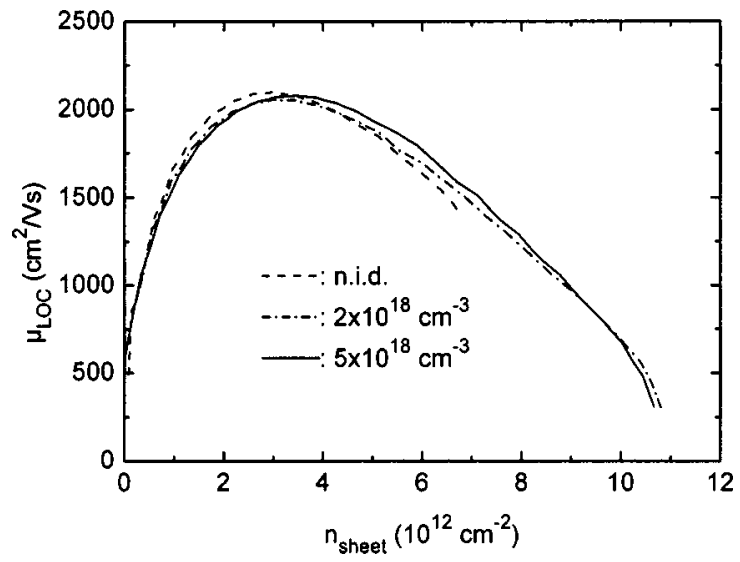

FIG. 3. Local drift mobility in intentionally undoped and doped AlGaN/ $\mathrm{GaN}$ heterostructures as a function of sheet concentration.

AIP license or copyright, see http://apl.aip.org/apl/copyright.jsp 
to $2100 \mathrm{~cm}^{2} / \mathrm{V} \mathrm{s}$ at $3 \times 10^{12} \mathrm{~cm}^{-2}$, and then decreases smoothly with a further increase of $n_{\text {sheet }}$. The mobility increase with the sheet carrier density is due to increased screening of carrier scattering by ionized impurities and dislocations in the 2DEG. ${ }^{5,12,13}$ Several authors ${ }^{6,13}$ explain the subsequent mobility reduction by the influence of the parallel low mobility channel at positive gate bias due to the carrier supply layer. However, our investigations show a mobility reduction long before the parallel channel is activated. In fact the gate capacitance remains constant during mobility decrease, and the decrease is even independent on the existence or the doping concentration of a carrier supply layer. The activation of the parallel channel in the carrier supply layer is also observed in our modulation-doped samples, but not until the sheet concentration exceeds $10^{13} \mathrm{~cm}^{-2}$. Ridley et al. ${ }^{12}$ have calculated the carrier density dependence of the mobility of a structure similar to ours. They find a mobility peak of $2000 \mathrm{~cm}^{2} / \mathrm{V} \mathrm{s}$ at $2 \times 10^{12} \mathrm{~cm}^{-2}$ carrier density. The mobility decrease at larger sheet concentrations is attributed to intersubband scattering as electrons begin to occupy the upper subbands in the potential well of the AlGaN/GaN heterointerface. The separation of the channel electrons from the carrier supply layer prevents electron scattering at the doping atoms that would reduce channel mobility of the doped samples.

In conclusion, we investigated the channel properties of intentionally-undoped and modulation-doped AlGaN/GaN HEMTs on $\mathrm{SiC}$ substrate. The average values of mobility and carrier density at $0 \mathrm{~V}$ gate voltage correspond with the results of conventional Hall effect measurements. The mobility as function of the sheet carrier density shows the same dependence for all samples. This means that the donor atoms in the carrier supply layer do not influence the mobility of the channel electrons in the 2DEG channel. The obtained results are in agreement with theoretical predictions by Ridley et $a{ }^{12}{ }^{12}$ They suggest that a carrier supply layer will not degrade the 2DEG transport properties of HEMT devices. On the other hand, this result supports our previous observation of better overall AlGaN/GaN HEMT performance using doped structure in comparison to undoped one. ${ }^{8}$

The authors thank J. Flynn and G. Brandes from ATMI Corporate, Danbury $\mathrm{CN}$, for providing samples on $\mathrm{SiC}$ substrates.

${ }^{1}$ L. F. Eastman, V. Tilak, V. Kaper, J. Smart, R. Thompson, B. Green, J. R. Shealy, and T. Prunty, Phys. Status Solidi A 194, 433 (2002).

${ }^{2}$ J. A. Bardwell, Y. Liu, H. Tang, J. B. Webb, S. J. Rolfe, and J. Lapointe, Electron. Lett. 39, 564 (2003).

${ }^{3}$ R. Gaska, M. S. Shur, A. D. Bykhovski, A. O. Orlov, and G. L. Snider, Appl. Phys. Lett. 74, 287 (1999).

${ }^{4}$ Z. Dziuba, J. Antoszewski, J. M. Dell, L. Faraone, P. Kozodoy, S. Keller, B. Keller, S. P. DenBars, and U. K. Mishra, J. Appl. Phys. 82, 2996 (1997).

${ }^{5}$ M. J. Uren, T. Martin, B. T. Hughes, K. P. Hilton, A. Wells, R. S. Balmer, D. C. Herbert, A. M. Keir, D. J. Wasslis, A. J. Pidduck, and M. Missous, Phys. Status Solidi A 194, 468 (2002).

${ }^{6}$ X. Z. Dang, P. M. Asbeck, E. T. Yu, G. J. Sullivan, M. Y. Chen, B. T. McDermott, K. S. Boutros, and J. M. Redwing, Appl. Phys. Lett. 74, 3890 (1999).

${ }^{7}$ D. C. Look, J. Appl. Phys. 57, 377 (1985).

${ }^{8}$ J. Bernát, M. Wolter, A. Fox, M. Marso, J. Flynn, G. Brandes, and P. Kordoš, Electron. Lett. 40, 78 (2004).

${ }^{9}$ M. Shur, GaAs Devices and Circuits (Plenum, New York, 1987), p. 578.

${ }^{10}$ K. Lee, M. S. Shur, T. J. Drummond, and H. Morkoç, IEEE Trans. Electron Devices ED-31, 29 (1994).

${ }^{11}$ D. C. Look, Electrical Characterization of GaAs Materials and Devices (Wiley, Chichester, 1989), p. 162.

${ }^{12}$ B. K. Ridley, B. E. Foutz, and L. F. Eastman, Phys. Rev. B 61, 16862 (2000).

${ }^{13}$ P. A. Ivanov, M. E. Levinshtein, G. Simin, X. Hu, J. Yang, M. A. Khan, S. L. Rumyantsev, M. S. Shur, and R. Gaska, Electron. Lett. 37, 1479 (2001). 